\title{
Proline Accumulation and AtP5CS2 Gene Activation Are Induced by Plant-Pathogen Incompatible Interactions in Arabidopsis
}

\author{
Georgina Fabro, ${ }^{1}$ Izabella Kovács, ${ }^{2}$ Valeria Pavet, ${ }^{1}$ László Szabados, ${ }^{2}$ and María E. Alvarez ${ }^{1}$ \\ ${ }^{1}$ CIQUIBIC-CONICET, Departamento de Química Biológica, Facultad de Ciencias Químicas Universidad Nacional \\ de Córdoba, 5000 Córdoba, Argentina; ${ }^{2}$ Institute Plant Biology, Biological Research Center, Szeged, Hungary
}

Submitted 22 September 2003. Accepted 17 November 2003.

\begin{abstract}
Accumulation of free L-proline (Pro) is a typical stress response incited by osmotic injuries in plants and microorganisms. Although the protective role of Pro in osmotic stress is not well understood, it is thought to function as compatible osmolyte or as a scavenger of reactive oxygen species (ROS). Here we show that, in Arabidopsis thaliana, Pro biosynthesis can be activated by incompatible plantpathogen interactions triggering a hypersensitive response (HR). Pro accumulates in leaf tissues treated with Pseudomonas syringae pv. tomato avirulent strains (avrRpt2 and avrRpm1) but remains unchanged in leaves infected with isogenic virulent bacteria. Incompatible interactions lead to transcriptional activation of AtP5CS2, but not AtP5CS1, encoding the rate limiting enzyme in Pro biosynthesis pyrroline-5-carboxylate synthase (P5CS). AtP5CS2:GUS and $A t P 5 C S 2: L U C$ transgenes were induced inside and around the HR lesions produced by avirulent Pseudomonas spp. in transgenic plants. Pro accumulation was faster and stronger when stimulated by avrRpm1 than by avrRpt2, and was compromised in the low-salicylic acid plants $N a h G$ and $e d s 5$ when signaled through the RPS2-dependent pathway. In addition, Pro content and AtP5CS2 expression were enhanced by ROS in wild-type plants, suggesting that ROS may function as an intermediate signal in AtP5CS2-mediated Pro accumulation.
\end{abstract}

Additional keyword: ProDH.

Higher plants can accumulate free L-proline (Pro) in response to different environmental stresses, such as drought and salinity (Delauney and Verma 1993; Rhodes et al. 1986), cold (Savouré et al. 1997; Xin and Browse 1998), or UV radiation (Saradhi et al. 1995). Pro accumulation is thought to function as a compatible osmolyte that stabilizes membranes and subcellular components (Delauney and Verma 1993; Rhodes et al. 1986), including the mitochondrial electron transport complex II (Hamilton and Heckathorn 2001). In addition, Pro is proposed to scavenge free radicals (Saradhi et al. 1995; Siripornadulsil et al. 2002; Smirnoff and Cumbes 1989) and to ameliorate shifts in redox potential by replenishment of the NADP+ supply (Delauney and Verma 1993; Hare and Cress 1997).

Corresponding author: M. E. Alvarez; Telephone: (+54) 0351 4334171; Fax: (+54) 0351 4334074; E-mail: malena @dqb.fcq.unc.edu.ar

L. Szabados and M. E. Alvarez contributed equally to this article.
A positive correlation between Pro accumulation and osmotic stress tolerance has been reported in several experimental systems (Gilmour et al. 2000; Nanjo et al. 1999). However, a stringent causal relationship between Pro accumulation and stress tolerance is not yet firmly established. Whereas some Pro-accumulating Arabidopsis mutants, such as eskimol, display enhanced stress tolerance (Xin and Browse 1998), others, such as sos 1 , are hypersensitive to stress (Liu and Zhu 1997). Moreover, Pro accumulation may result in toxicity under specific metabolic conditions (Hellman et al. 2000; Mani et al. 2002).

In higher plants, Pro is synthesized from glutamate or ornithine and the first pathway is considered to be dominant under stress conditions (Delauney and Verma 1993; Roosens et al. 1999). In the glutamate pathway, Pro biosynthesis is initiated through phosphorylation and reduction of glutamate to glutamyl-5-semialdehyde (G5SA) by the bifunctional of $\Delta^{1}$ pyrroline-5-carboxylate synthase (P5CS). G5SA is converted to Pro through pyrroline-5-carboxylate (P5C) by the $\Delta^{1}$-pyrroline-carboxylate reductase (P5CR) (Delauney and Verma 1993; Hu et al. 1992). The $\gamma$-glutamyl kinase activity of P5CS represents the rate-limiting step in this pathway and is sensitive to feedback inhibition by Pro (Roosens et al. 1999; Zhang et al. 1995). Pro catabolism involves its oxidization into glutamate by a two-step reaction including two mitochondrial enzymes, proline dehydrogenase (ProDH) and P5C-dehydrogenase (P5CDH) (Deuschle et al. 2001; Kiyosue et al. 1996; Verbruggen et al. 1996).

In Arabidopsis thaliana, drought and salt stress differently activate the expression of two related P5CS genes: AtP5CS1 (At2g39800) and AtP5CS2 (At3g55610). AtP5CS1 is responsible mainly for Pro accumulation during salt and drought stress. Expression of AtP5CS1 is activated by an ABA-dependent signal transduction pathway and modulated by light and brassinosteroid (Ábrahám et al. 2003; Savouré et al. 1997; Strizhov et al. 1997; Yoshiba et al. 1995). AtP5CS2 transcription is slightly elevated by salt stress (Strizhov et al. 1997) and induced by cold through the CBF3 transcription factor (Gilmour et al. 2000). Pro catabolism is controlled by ProDH (At3g30775), whose transcription is activated by enhanced Pro levels and is repressed under osmotic stress conditions (Kiyosue et al. 1996; Verbruggen et al. 1996).

Infections with virulent races of Pseudomonas syringae lead to the development of disease in Arabidopsis plants (Whalen et al. 1991). In contrast, the perception of avirulent races of the bacterium stimulates a complex set of plant defenses leading to disease resistance. Thus, $A$. thaliana Col-0 plants expressing the $R P S 2$ and $R P M 1$ resistance $(R)$ genes recognize, directly 
or indirectly, the bacterial avirulent products encoded by the avrRpt 2 or avrRpml genes, respectively, activating gene-forgene mediated disease resistance and hypersensitive response (HR) (Bent et al. 1994; Grant et al. 1995; Mindrinos et al. 1994).

Cross-talks between abiotic and biotic defense programs have been suggested (Genoud and Métraux 1999; Singh et al. 2002). Early common protective responses include alterations at plasma membrane ion channels, up-regulation of the oxidative metabolism producing reactive oxygen species (ROS), and calcium influx (Lamb and Dixon 1997; Mittler 2002). The importance of salicylic acid (SA) in pathogen-induced disease

A

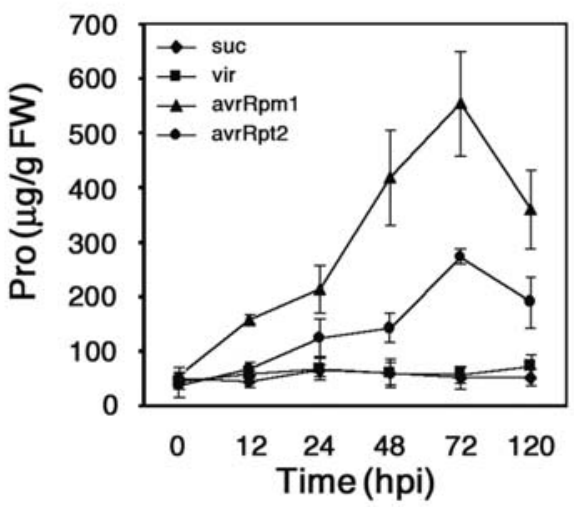

B
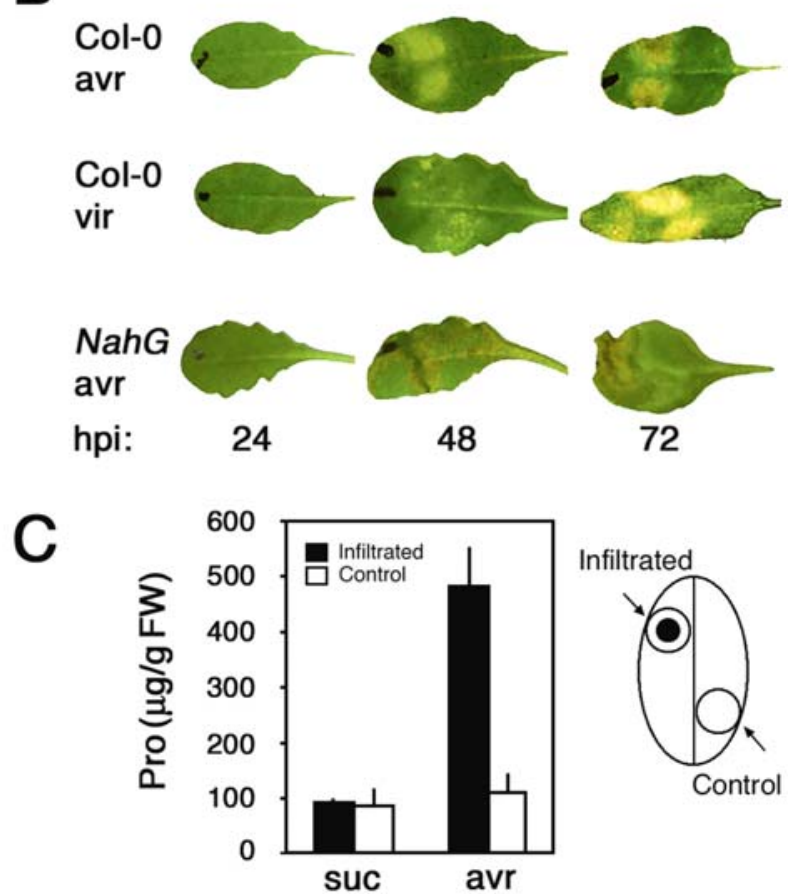

Fig. 1. Free L-proline (Pro) accumulates in Arabidopsis leaves treated with avirulent races of Pseudomonas. A, Pro levels in entire leaves, infiltrated at two spot sites with $0.4 \mathrm{M}$ sucrose (suc), or bacterial suspensions $\left(5 \times 10^{6} \mathrm{CFU} / \mathrm{ml}\right)$ of virulent (vir) or avirulent (avrRpml and avrRpt2) races of Pseudomonas syringae pv. tomato. Values represent the average \pm standard deviation of three independent experiments including at least five leaves per treatment. B, Typical leaf symptoms developed by wild-type and NahG plants after infiltration of the avrRpt 2 bacterial suspensions used for Pro determination; hpi = hours postinfiltration. $\mathbf{C}$, Local Pro accumulation in avrRpt2-treated Arabidopsis leaves. Bacterial suspensions were infiltrated at spots of 3 to $4 \mathrm{~mm}$ in diameter. At $72 \mathrm{hpi}$, 9- to 12-mm-diameter discs containing infiltrated (central) or noninfiltrated (periphery) areas were excised from the same leaves for Pro determination as indicated in the diagram. resistance and hypersensitive cell death is well documented (Alvarez 2000; Delaney et al. 1994; Gaffney et al. 1993). Through the potentiation of oxidative burst, SA can control both biotic and abiotic defense programs (Borsani et al. 2001; Shirasu et al. 1997).

Using genetically defined pathosystems involving $A$. thaliana and $P$. syringae to either activate gene-for-gene disease resistance (avirulent pathogen; incompatible interaction) or produce disease (virulent pathogen; compatible interaction), we evaluated whether Pro metabolism is altered under biotic stress conditions. Here we demonstrate that Pro is accumulated specifically in Arabidopsis leaf tissues developing incompatible interactions involving avrRpt2:RPS2 or avrRpm1:RPM1 recognition and leading to HR. This response involves the transcriptional activation of AtP5CS2 but not AtP5CS1, and it can be induced by ROS. Moreover, Pro accumulation signaled through the RPS2 pathway depends on SA as well.

\section{RESULTS}

Pro accumulates in Arabidopsis tissues developing incompatible interactions.

Expanded leaves of $A$. thaliana Col-0 plants were infiltrated at two discrete sites with approximately $10 \mu \mathrm{l}$ of bacterial suspensions $\left(5 \times 10^{6} \mathrm{CFU} / \mathrm{ml}\right)$ of isogenic virulent (vir) and avirulent (avrRpt2 and avrRpml) races of $P$. syringae pv. tomato DC3000 or dilution solution (mock inoculation). Bacteria were diluted in $0.4 \mathrm{M}$ sucrose instead of the $10 \mathrm{mM} \mathrm{MgCl}$ classically used for such purpose (Alvarez et al. 1998; Nawrath and Métraux 1999). Infiltration of $10 \mathrm{mM} \mathrm{MgCl} 2$ alone increased the osmotic potential of leaf tissues from $3.71 \pm 0.75 \mathrm{Mpa}$ to $-1.89 \pm 0.11 \mathrm{Mpa}$ and produced a slight increase in Pro content in those tissues (1.5-fold; 1 to $3 \mathrm{~h}$ postinfection [hpi]) (data not shown). In contrast, infiltration of 0.4 $\mathrm{M}$ sucrose altered neither the osmotic potential $(-3.15 \pm 0.58$ Mpa) nor the Pro levels in inoculated tissues, when tested at 24,48 , and $72 \mathrm{~h}$ after infiltration. Pathogens infiltrated in this solution produced the typical HR (avrRpt 2 and avrRpml) or disease (vir) symptoms in A. thaliana Col-0 plants (Alvarez et al. 1998; Bent et al. 1994; Grant et al. 1995; Mindrinos et al. 1994) (Fig. 1B). Moreover, sucrose infiltration did not modify the basal expression pattern of Pro-metabolic genes at these time points (Fig. 2).

The average of Pro levels of individual untreated leaves from 5- to 6-week-old plants was $46.5 \pm 15.6 \mu \mathrm{g}$ per $\mathrm{g}$ of fresh weight (Fig. 1A). Pro levels started to increase at $12 \mathrm{hpi}$ with avrRpml and at 24 hpi with avrRpt2. Such Pro enhancement preceded the macroscopic detection of net HR lesions in the challenged tissues, which became visible at 24 hpi with avrRpm1 (data not shown) or 48 hpi with avrRpt2 (Fig. 1B). The $a v r$-treated tissues accumulated Pro until 72 hpi, reaching 7- and 12-fold increase over the basal values when inoculated with avrRpt 2 or avrRpm1, respectively. By 5 days postinfection (dpi), both responses declined and leaves displayed net signs of cellular collapse (data not shown). In contrast, Pro levels remained essentially unchanged until $5 \mathrm{dpi}$ in plants treated with either vir or sucrose (Fig. 1A). These results indicated that Pro accumulation is elicited specifically upon R or avirulence (avr) recognition and that kinetic and quantitative differences are displayed when the response is achieved by stimulation of the RPM1- or RPS2-dependent pathways.

Local differences in Pro content were analyzed at and near the infiltration sites for avrRpt 2 treatments. Bacterial suspensions or $0.4 \mathrm{M}$ sucrose were infiltrated as spots of 3 to $4 \mathrm{~mm}$ in diameter. Infiltrated and noninfiltrated tissues from the same leaves were excised separately in 9- to 12-mm-diameter 
leaf discs and used for Pro quantification. At 72 hpi, only leaf samples taken from the avrRpt2-infiltrated areas displayed a net increase in Pro content, whereas the noninfiltrated tissues had essentially unaltered Pro concentration (Fig. 1C). The virand sucrose-inoculated samples showed no differences in Pro content at 72 hpi (data not shown).

\section{Expression of Pro metabolism genes in pathogen-inoculated tissues.}

Pro levels are tightly controlled by biosynthetic and catabolic activities. To monitor the Pro biosynthetic and catabolic pathways during pathogen infection, we analyzed the transcription levels of the A. thaliana AtP5CS1 and AtP5CS2 genes controlling the glutamate biosynthetic pathway, and the ProDH and $P 5 C D H$ genes participating in Pro degradation. Northern blot experiments revealed that AtP5CS2, but not AtP5CS1, was activated by avrRpt2 inoculation (Fig. 2) and reached up to eightfold increased levels at $72 \mathrm{hpi}$. Such AtP5CS2 activation paralleled Pro accumulation in avrRpt2treated tissues (Fig. 1A). In contrast, AtP5CS2 expression was almost unaffected by vir or mock inoculations (Fig. 2). On the other hand, AtP5CS1 expression was not altered by any of these treatments (Fig. 2). Upon avrRpt2 inoculation, ProDH transcript levels increased 3 to 4 times from 0 to $72 \mathrm{hpi}$, while no alterations were observed in vir- or mock-inoculated leaves (Fig. 2). In addition, P5CDH expression was not modified in vir-, avrRpt2-, or sucrose-infiltrated Arabidopsis leaves (Fig. 2). These results suggest that Pro accumulation in HR developing tissues principally derives from the induction of AtP5CS2.

In order to localize AtP5CS activation in response to $P$. syringae pv. tomato inoculation, transgenic Arabidopsis plants expressing the $\beta$-glucuronidase $(G U S)$ or firefly luciferase $(L U C)$ reporter genes under the control of AtP5CS1 and AtP5CS2 promoters (Ábrahám et al. 2003) were infiltrated by vir and avrRpt2. The spatiotemporal pattern of GUS and LUC activities were analyzed along with development of disease or HR. Very low basal GUS activity was detected in naive AtP5CS2:GUS plants (Fig. 3A), which is consistent with previous observations about the low abundance of AtP5CS2 transcripts in leaf tissues (Strizhov et al. 1997). AtP5CS2:GUS expression was activated in leaves infiltrated with the avirulent pathogen (Fig. 3A). This response initially was detected by 24
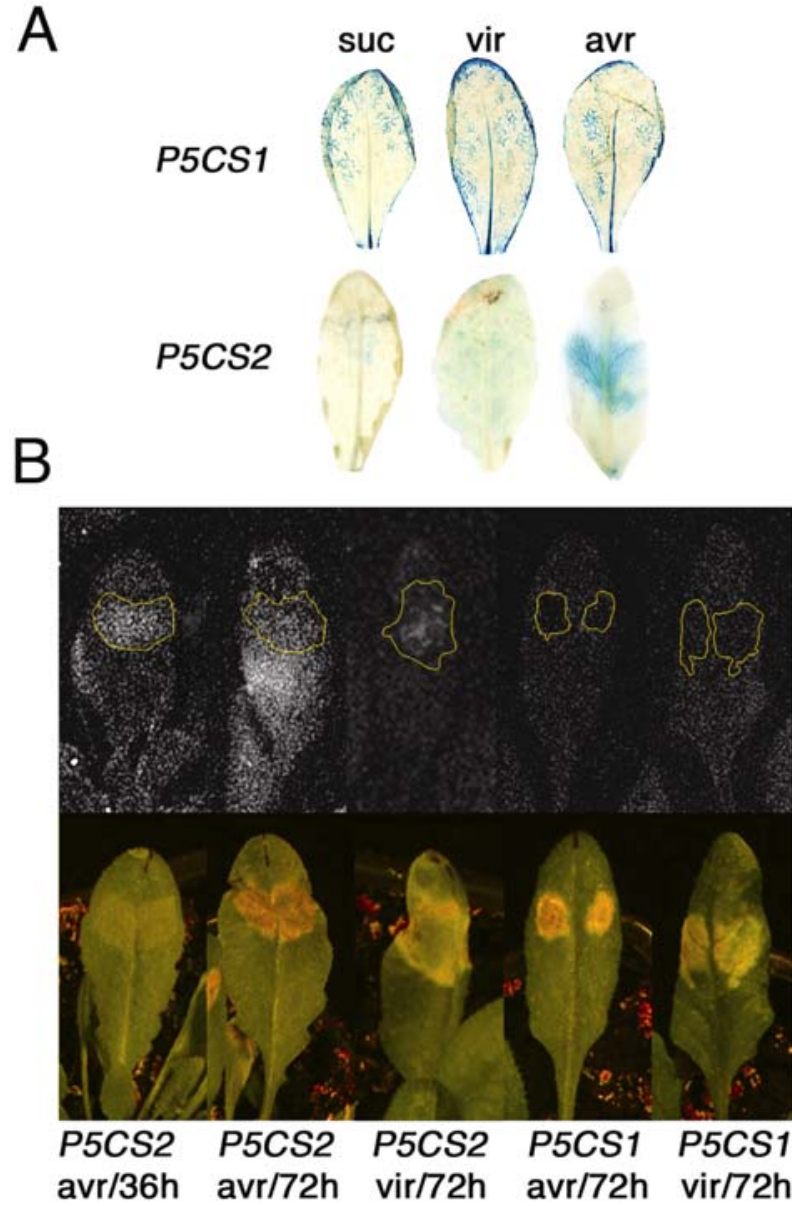

Fig. 3. Spatiotemporal pattern of AtP5CS1 and AtP5CS2 expression in hypersensitive response. Transgenic Arabidopsis plants containing the $A t P 5 C S 1$ and AtP5CS2 gene promoters fused to the $\beta$-glucuronidase $(G U S)$ or firefly luciferase $(L U C)$ reporter genes were inoculated at two spot sites with $0.4 \mathrm{M}$ sucrose (suc), or bacterial suspensions $\left(5 \times 10^{6}\right.$ $\mathrm{CFU} / \mathrm{ml}$ ) of virulent (vir) or avirulent (avrRpml and avrRpt2) races of Pseudomonas syringae pv. tomato. A, GUS activity was detected by Xgluc histochemical staining at $48 \mathrm{~h}$ postinfiltration. B, LUC expression was monitored by luminescence imaging at the indicated times after infiltration. Yellow lines indicate the borders of lesions.
A
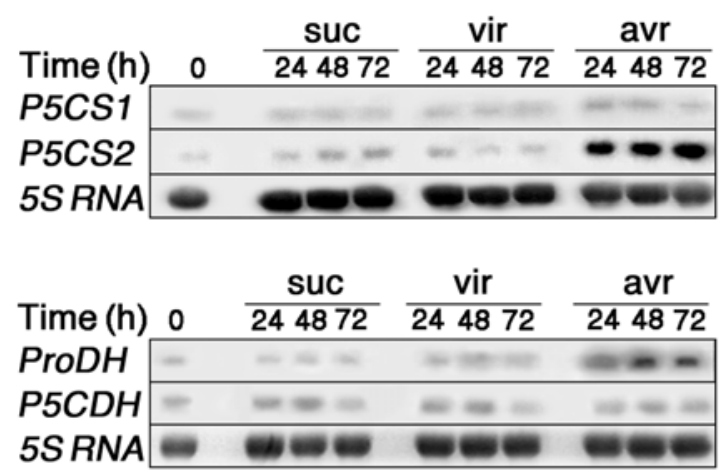

B

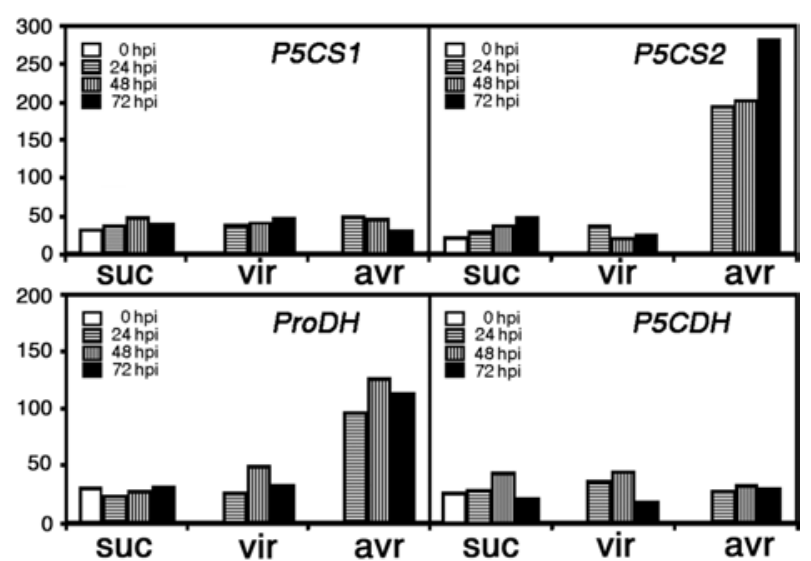

Fig. 2. AtP5CS2 and ProDH are activated by infiltration of avirulent bacteria. Leaves were infiltrated at two spot sites with $0.4 \mathrm{M}$ sucrose (suc), or bacterial suspensions $\left(5 \times 10^{6} \mathrm{CFU} / \mathrm{ml}\right)$ of virulent (vir) or avirulent (avrRpml and avrRpt2) races of Pseudomonas syringae pv. tomato. A, Northern blot analysis of genes AtP5CS1, AtP5CS2, ProDH, and P5CDH on total RNA samples isolated from avrRpt2-infiltrated leaves. Hybridization with 5S RNA was used as loading control. B, Relative values of the AtP5CS1, AtP5CS2, ProDH, and P5CDH transcript levels detected by Northern hybridization. Values were normalized to $5 \mathrm{~S}$ rRNA content on each well. 
hpi at the inoculation sites and enhanced by 48 and $72 \mathrm{hpi}$, when it often was visible in neighboring tissues surrounding the infiltration sites. The vir infiltration occasionally led to AtP5CS2:GUS activation, whereas mock inoculations did not activate AtP5CS2:GUS over this period. Identical treatments were performed with transgenic plants carrying the AtP5CS1:GUS construct, whose expression was not induced by any $P$. syringae pv. tomato inoculation (Fig. 3A).

AtP5CS1 and AtP5CS2 promoter activities also were monitored in living tissues of transgenic plants expressing the firefly luciferase gene fusions, where images of luminescence could be compared directly with the development of symptoms (Fig. 3B). LUC activity was first detected in avrRpt2treated leaves of AtP5CS2:LUC plants at $24 \mathrm{hpi}$ at the center of the infiltration site. One or two days later, LUC activity usually was localized in infiltrated tissues or sometimes shifted to
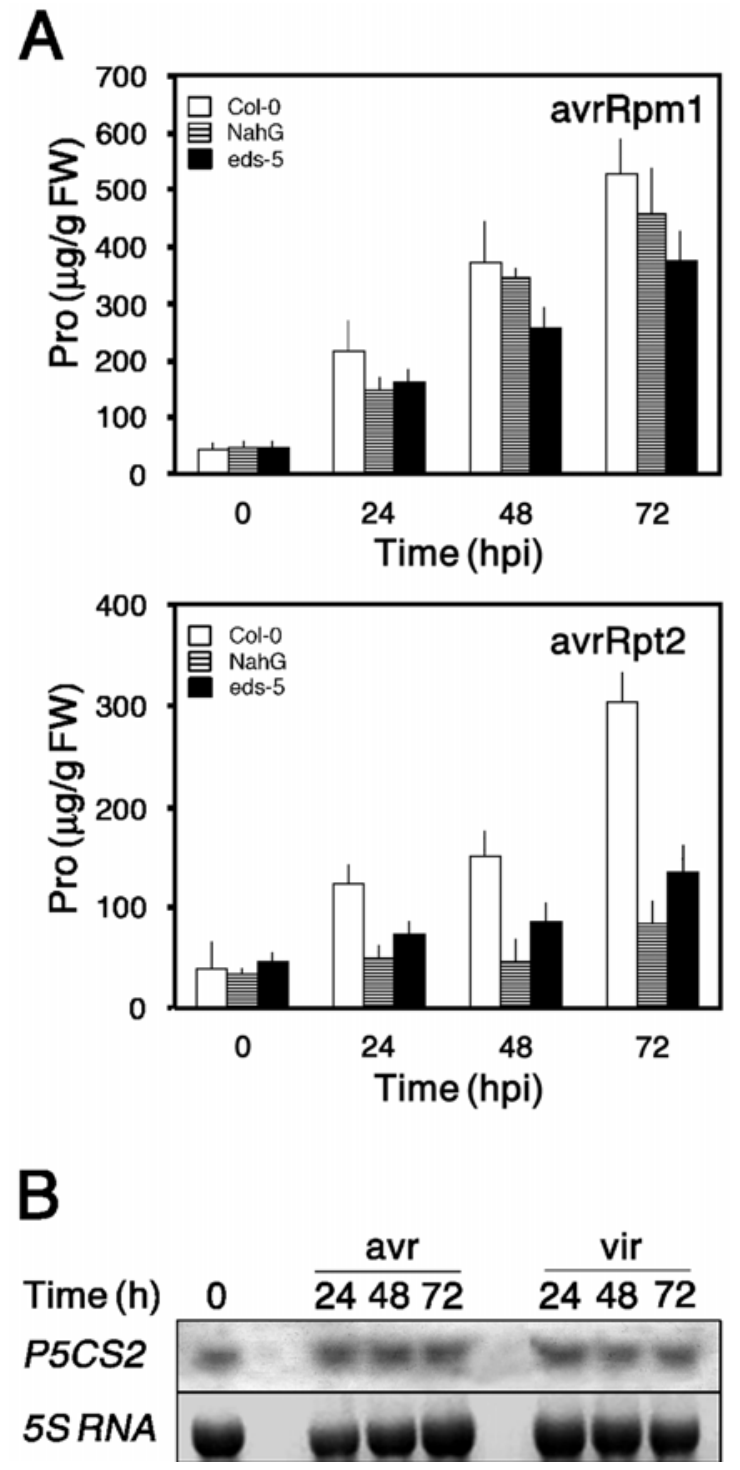

Fig. 4. Requirement of salicylic acid (SA) for L-proline (Pro) accumulation induced through the RPM1- or RPS2-dependent pathways. A, Pro accumulation in entire leaves of wild-type, NahG, and eds5 Arabidopsis plants infiltrated with avrRpm1 (upper panel) or avrRpt2 (lower panel). Leaves were infiltrated at two spot sites with $0.4 \mathrm{M}$ sucrose, or bacterial suspensions $\left(5 \times 10^{6} \mathrm{CFU} / \mathrm{ml}\right)$ of virulent (vir) or avirulent (avr) races of $P$. syringae pv. tomato. Samples were collected at the indicated times. hpi $=$ hours postinfiltration. Values represent the average of three independent experiments. B, Northern blot assays developed with AtP5CS2 probes in $N a h G$ leaves infected with vir or avrRpt2 (avr) bacteria. regions surrounding the HR lesions (Fig. 3B). LUC activity was not detected far from the infiltrated regions of leaves treated with avrRpt 2 .

AtP5CS1:LUC transgenic plants had no increased luminescence upon bacterial inoculation. These plants occasionally showed LUC activity in necrotic, dry, or mechanically damaged tissues that had no correlation with the type of infiltration. These results confirmed that AtP5CS2 is the major gene activated at the transcriptional level by plant-pathogen incompatible interactions around the infection site.

\section{SA and ROS modulate Pro accumulation.}

SA is known to be required for the proper activation of local and systemic pathogen-induced defense networks (Alvarez 2000). Transgenic NahG plants are depleted in SA by overexpression of salicylate hydroxylase, which oxidizes SA into catechol. These plants are more susceptible to pathogen attack and are unable to develop confined HR lesions or activate systemic acquired resistance (Alvarez 2000; Delaney et al. 1994; Gaffney et al. 1993) (Fig. 1B). The eds5 mutant fails to accumulate SA after pathogen attack and EDS5, homologue to multidrug and toxin extrusion transporters, is suspected to be involved in SA biosynthesis (Nawrath and Métraux 1999). In order to test whether $a v r$-induced Pro enhancement depends on SA accumulation, Pro levels were quantified in avrRpt2- or avrRpml-infiltrated $N a h G$ and $e d s 5$ plants, which developed cell death in response to these treatments (Fig 1C; data not shown). Similar basal Pro levels were detected in leaf tissues of $N a h G, e d s 5$, and wild-type plants (Fig. 4A). NahG and $e d s 5$ plants were compromised in Pro accumulation elicited by avrRpt 2 , reaching only $28 \%(N a h G)$ or $44 \%$ (eds5) of the Pro levels found in wild-type plants at 72 hpi (Fig. 4A). AtP5CS2 expression was not induced in $N a h G$ plants upon perception of avrRpt2, as happened in wild-type plants (Fig. 2), and neither was this gene activated in response to the vir pathogen (Fig. $4 \mathrm{~B})$. On the other hand, AtP5CS1 expression was not modified by avrRpt2, vir, or mock inoculations in NahG plants (data not shown). Interestingly, $N a h G$ and $e d s 5$ plants only weakly suppressed the Pro accumulation activated by avrRpml recognition, containing $88 \%(N a h G)$ and $72 \%$ (eds5) of the levels detected in wild-type plants at 72 hpi (Fig. 4A). These results indicated that the stimulation of a unique $\mathrm{R}$ gene-dependent pathway activates similar responses in both low-SA plants and that each pathway is differently affected by SA deficiency to signal Pro accumulation.

The requirement of SA for producing a sustained enhancement of Pro levels and AtP5CS2 activation in RPS2-mediated incompatible interactions prompted us to analyze whether SA itself induced such responses. Wild-type plant leaves were infiltrated with 10,50 , and $150 \mu \mathrm{M}$ SA and Pro content was determined at $0,3,6$, and 9 hpi. The SA concentrations utilized in these treatments were in the same range found in $A$. thaliana leaf tissues developing incompatible interactions ( $\mathrm{Bi}$ et al. 1995). A transient Pro increase (fourfold) was detected at 6 hpi with 50 and $150 \mu \mathrm{M} \mathrm{SA}$, but this response was not sustained by 9 hpi (data not shown). Thus, exogenous SA treatments were not able to reproduce the Pro accumulation effect generated by avrRpt 2 infiltration. When AtP5CS2:GUS or AtP5CS2:LUC plants were infiltrated with $150 \mu \mathrm{M}$ SA, only faint or background activity of the reporter genes was detected in treated leaves at $7 \mathrm{hpi}$ (Fig. 5C and D).

SA potentiates the oxidative burst and hypersensitive cell death in genetically defined disease resistance (Lamb and Dixon 1997; Shirasu et al. 1997). Therefore, we evaluated whether externally generated ROS can modulate Pro accumulation. Arabidopsis leaves were infiltrated locally with the enzymatic mix of xanthine and xanthine oxidase (X-XO) that 
produces superoxide anion in situ (Jabs et al. 1996). Upon X$\mathrm{XO}$ treatment, a progressive and sustained increase in Pro content occurred during the initial 9 hpi (Fig. 5A). Moreover, positive correlation was found between Pro levels and concentrations of xanthine oxidase enzyme employed in infiltration (Fig. 5B). These results indicated that leaf tissues can sensitively increase their Pro content upon ROS accumulation. Histochemical GUS assay detected AtP5CS2:GUS activity in leaf tissues infiltrated with X-XO (Fig. 5C). Similar results were obtained with transgenic AtP5CS2:LUC plants, where luminescence could be detected at X-XO infiltration sites (Fig. 5D). These results indicated that ROS can mediate the activation of AtP5CS2 promoter in situ and eventually function during biotic stress.

\section{DISCUSSION}

Pro content increases in Arabidopsis leaf tissues developing incompatible interactions with $P$. syringae pv. tomato.

In this work, we describe that Pro accumulation, a typical plant osmotic stress response, also occurs in response to biotic stress. Our results demonstrate that free Pro content increases in Arabidopsis leaf tissues that activate an HR upon recognition of avirulent races of $P$. syringae pv. tomato (avrRpt2 and avrRpm1). Pro accumulation is detected as early as 12 to $24 \mathrm{~h}$ after treatment with avirulent bacteria, and the magnitude of increase (7- to 12-fold at the third day) is comparable with that reported for abiotic stresses (Delauney and Verma 1993; Kishor et al. 1995; Roosens et al. 1999). These values are not substantially exceeded by transgenic plants designed to overproduce Pro by manipulation of Pro metabolic genes (Nanjo et al. 1999) or by some of the Arabidopsis mutants that constitutively accumulate this imino acid (Xin and Browse 1998). On the other hand, Pro content was not enhanced in Arabidopsis leaves developing necrosis in response to virulent Pseudomonas infection.

Biosynthesis and catabolism of Pro are tightly regulated processes involving two key enzymatic activities, P5CS and ProDH (Delauney and Verma 1993, Hare and Cress 1997). In A. thaliana, P5CS contributes to Pro accumulation under abiotic stress conditions mainly through ABA-dependent transcriptional activation of the AtP5CS1 gene (Savouré et al. 1997; Strizhov et al. 1997; Yoshiba et al. 1995). ProDH, which controls Pro catabolism, becomes transcriptionally activated by enhanced Pro levels, but can be repressed during osmotic stress, permitting Pro accumulation under such conditions (Kiyosue et al. 1996; Verbruggen et al. 1996). Our data showed that transcription of AtP5CS2 and, to a lesser extent, ProDH, was induced in response to the avirulent but not to the virulent pathogens, whereas AtP5CS1 and P5CDH transcription did not change under these conditions (Fig. 2).

The avrRpt2-induced Pro accumulation correlates with the increase in AtP5CS2 transcript levels during 72 hpi, suggesting enhancement of the biosynthetic pathway in the infected leaves through AtP5CS2 gene activation. ProDH expression also is induced over this period (Fig. 2); however, the continuous rise in Pro content in avr-treated tissues (Fig. 1) would suggests that Pro biosynthesis is more effective than Pro catabolism at this stage. In avrRpt2-infiltrated leaves, ProDH transcription probably was activated by the accumulating Pro, as happens in nonstressed plants in the absence of osmotic repression (Kiyosue et al. 1996; Verbruggen et al. 1996). Therefore, our findings suggest that, under biotic stress conditions, Pro levels are controlled by transcriptional regulation of AtP5CS2 and ProDH genes. Further studies will be required to reveal possible modifications in the activity of P5CS and ProDH enzymes in response to pathogen attack.
ABA-responsive AtP5CS1 is the major gene induced under osmotic stress (Strizhov et al. 1997; Yoshiba et al. 1995) but does not seem to be responsible for the increased biosynthesis of Pro in avrRpt2-treated plants. AtP5CS2 is moderately activated by cold or osmotic stress and previously has been associated with cell division events and housekeeping functions (Ábrahám et al. 2003; Gilmour et al. 2000; Strizhov et al. 1997). Therefore, specific activation of AtP5CS2 during plantpathogen incompatible interaction describes a novel function of this gene in plant defense.

Pro is considered a compatible osmolyte in osmotic stress conditions (Delauney and Verma 1993), and has been suggested to function as a scavenger of hydroxyl radicals, controlling redox homeostasis as well (Hare and Cress 1997; Siripornadulsil et al. 2002; Smirnoff and Cumbes 1989). Our results show that
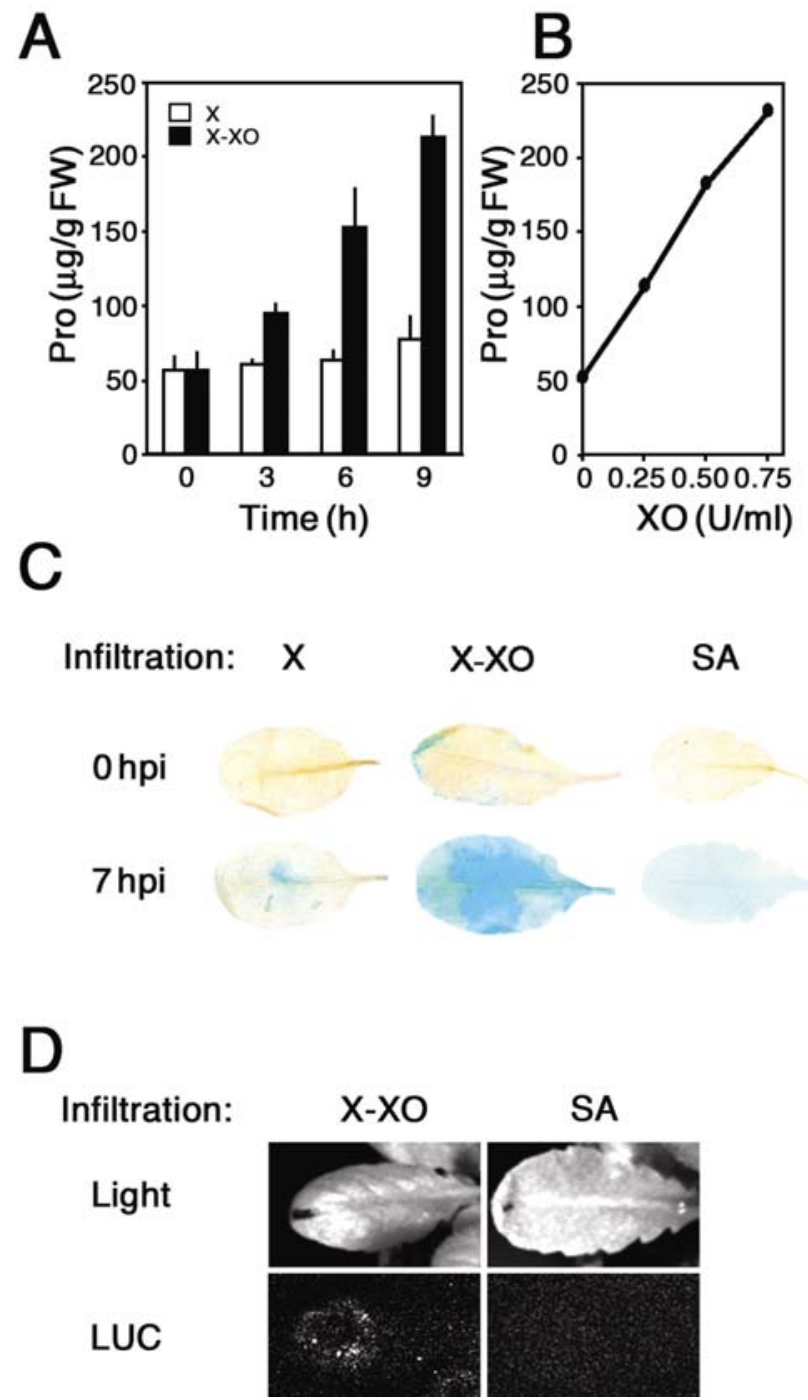

Fig. 5. Reactive oxygen species accumulation mediates L-proline (Pro) enhancement and AtP5CS2 activation. A, Pro accumulation in entire Arabidopsis leaves locally infiltrated either with $1 \mathrm{mM}$ xanthine (X), or 1 $\mathrm{mM}$ xanthine and xanthine oxidase $(\mathrm{X}-\mathrm{XO})$ at $0.5 \mathrm{U} / \mathrm{ml}$. B, Lineal dependence of Pro response on a concentration range of xanthine oxidase able to signal hypersensitive cell death on Arabidopsis leaves (Jabs et al. 1996). Pro levels were measured at $9 \mathrm{~h}$ postinfiltration. C, Histochemical detection of $\beta$-glucuronidase (GUS) activity in AtP5CS2:GUS Arabidopsis leaves locally infiltrated with $1 \mathrm{mM}$ xanthine (X), $1 \mathrm{mM}$ xanthine and xanthine oxidase $(\mathrm{X}-\mathrm{XO})$ at $0.5 \mathrm{U} / \mathrm{ml}$, or $150 \mu \mathrm{M}$ salicylic acid (SA). GUS activity was analyzed at the indicated times. hpi = hours postinfiltration. D, Luciferase imaging of transgenic AtP5CS2:LUC Arabidopsis leaves treated with $\mathrm{X}-\mathrm{XO}$ or SA as described above and analyzed at $7 \mathrm{hpi}$. 
Pro accumulation occurs in and around the HR lesion (Fig. 1C). Scavenger properties of Pro may have importance in protecting pathogen-infected plant tissues once increased amount of ROS are generated. In HR-developing tissues, only AtP5CS2 and ProDH transcript levels were enhanced, suggesting that Pro biosynthetic and catabolic pathways are induced in the absence of $\mathrm{P5CDH}$ activation. Enhanced Pro turnover can lead to P5C accumulation when ProDH and P5CDH activities are uncoupled (Deuschle et al. 2001; Hellmann et al. 2000). Interconversion of P5C and Pro can influence NADP+ or NADPH levels (Hare and Cress 1997) and, therefore, might influence the extension of oxidative damage in HR-developing tissues. Moreover, P5C could activate apoptotic cell death under certain circumstances (Hellmann et al. 2000; Maxwell and Davis 2000). Further experiments are required to investigate putative functions of Pro and $\mathrm{P} 5 \mathrm{C}$ in biotic stresses leading to HR development.

\section{Plant signals involved in Pro accumulation and AtP5CS2 activation.}

A substantial accumulation of Pro was detected from 12 to 24 hpi with $P$. syringae pv. tomato avirulent pathogens that activate either the RPM1- or the RPS2-dependent defense pathways (Fig. 1A). Pro increase occurs in strict dependence on $\mathrm{R}$ and avr recognition for at least 5 days after pathogen inoculation (Fig. 1A). This response is earlier and more strongly achieved when it is induced by the RPM1 pathway. Likewise, gene expression profile changes and HR development are faster and more strongly induced by the RMP1 compared with the RPS2 pathway (Ritter and Dangl 1996; Tao et al. 2003). Pro accumulation may result from the activation of still unknown signaling components common to both defense cascades, which are proposed to share major signal transduction pathways (Tao et al. 2003).

Thus, up-regulation of Pro biosynthesis may require specific signaling factors of the RPM1- or RPS2-dependent pathways leading to disease resistance and HR. It is known that RPM1induced defenses are less affected by SA deficiency than those signaled through RPS2. NahG plants depleted in SA reduce more severely the RPS2- than RPM1-mediated resistance and development of hypersensitive cell death (Rate and Greenberg 2001; Tao et al. 2003). Similarly, R-gene mediated resistance, hypersensitive cell death, and global gene expression changes are reduced in a stronger manner for the RPS 2 pathway in the $n d r l$ mutant which is impaired in pathogen-induced SA accumulation (Tao et al. 2003). Therefore, we characterized features of RPM1- and RPS2-mediated Pro accumulation in two types of SA-deficient plant, the transgenic NahG line and the eds 5 mutant. In these plants, Pro accumulation clearly was reduced when generated via RPS2 but almost unaffected for its RPM1-dependent activation. Thus, Pro accumulation displays similar differences in SA sensitivity to the classical defense responses described above, suggesting that it may involve common signaling components.

Pro accumulation was similarly reduced in NahG and eds5 plants during RPS2-dependent activation (Fig. 4). Thus, irrespective of whether lack of SA is caused by impaired SA biosynthesis in eds5 (Nawrath and Métraux 1999) or by SA degradation in NahG (Delaney et al. 1994), reduction of RPS2dependent Pro accumulation in both plants must result from SA deficiency. Some NahG phenotypes were, in contrast, proposed to be caused by the accumulation of catechol instead of SA deficit (van Wees and Glazebrook 2003).

Our results showed that exogenous SA triggers only a limited accumulation of Pro and no or only weak activation of AtP5CS2 in wild-type plants (Fig. 5C and D). These findings suggest that, during induction of the RPS2-mediated re- sponses, $\mathrm{SA}$ is important but not sufficient to activate AtP5CS2 transcription and Pro enhancement. The signal flow for RPS2mediated defenses could be weaker and probably more sensitive to SA than that involving RPM1 (Tao et al. 2003). SA might function then by activating early steps in RPS2-dependent responses but requiring additional signals to maintain enhanced Pro levels. Transcriptional activation of AtP5CS2 by avrRpt 2 mainly occurs in and around the HR lesion (Fig. 3), where SA may reach its higher levels in the challenged leaves (Enyedi et al. 1992). Further studies will be required to elucidate whether SA locally contributes to activate AtP5CS2mediated Pro enhancement in HR.

The requirement of SA for proper activation of local defenses and HR has been extensively characterized (Alvarez 2000; Delaney et al. 1994). SA can potentiate, among other HR components, the early development of the oxidative burst preceding hypersensitive cell death (Shirasu et al. 1997). Looking for SA-dependent HR components that may contribute to signal Pro accumulation in response to avrRpt2, we evaluated the effect of ROS. We found that both Pro levels and AtP5CS2 promoter activity were enhanced by an external treatment generating superoxide anion in Arabidopsis leaves. Thus, the localized oxidative burst triggered at $a v r$-treated tissues may positively modulate AtP5CS2 activation and Pro accumulation. Generation of ROS is a common plant response to pathogen infection and to different adverse environmental conditions such as high light, UV-B irradiation, osmotic stress, soil salinity, cold, or high temperatures (Lamb and Dixon 1997; Shinozaki and Yamaguchi-Shinozaki 2000). ROS levels in photosynthetic tissues are controlled by SA during salt and osmotic stresses (Borsani et al. 2001). Therefore, common elements of pathogen defense and abiotic stress responses can be modulated by ROS.

Differences in transcriptional regulation of AtP5CS1 and AtP5CS2 suggest that alternative mechanisms regulate Pro biosynthesis in biotic and abiotic stress conditions. Although osmotic stress leads to AtP5CS1 induction and ProDH repression through an ABA-mediated signal transduction pathway, incompatible pathogen interactions result in SA-dependent or independent AtP5CS2 activation, probably involving ROS as intermediate signal.

\section{MATERIALS AND METHODS}

\section{Plant material.}

A. thaliana wild-type, eds5 (Nawrath and Métraux 1999), and NahG (Delaney et al. 1994) plants of the Columbia ecotype (Col-0) (genotype RPS2/RPS2 and RPM1/RPM1) were used in this study. Promoter analysis was performed with $A$. thaliana Col-0 transgenic plants carrying the $2.5-$ or $1.8-\mathrm{kb}$ promoter fragments of AtP5CS1 or AtP5CS2 genes, respectively, fused to the uidA (GUS) (Ábrahám et al. 2003) or firefly luciferase $(L U C)$ reporter genes. Plants were maintained in growth chambers under a photoperiod with $12 \mathrm{~h}$ of light and $12 \mathrm{~h}$ of dark at $22^{\circ} \mathrm{C}$ and watered every 2 days.

\section{Plant infiltration.}

Plants were used for infiltration at the age of 5 to 6 weeks. The virulent strain $P$. syringae pv. tomato DC3000 (Whalen et al. 1991) and the isogenic avirulent races avrRpt2 (Innes et al. 1993) and avrRpml (Ritter and Dangl 1996) were used for bacterial infiltration. Bacteria were grown overnight in King's $B$ medium supplemented with antibiotics and further used to infiltrate fully expanded leaves at a concentration of $5 \times 10^{6}$ $\mathrm{CFU} / \mathrm{ml}$ as described (Alvarez et al. 1998). Plants developing net HR lesions (avr) or disease symptoms (vir) were selected for further studies. To generate superoxide anion accumulation 
into intercellular spaces of leaf tissues, a mix of xanthine (1 $\mathrm{mM})$ and xanthine oxidase $(0.25$ to $0.75 \mathrm{U} / \mathrm{ml})$ was utilized as described (Jabs et al. 1996). Experiments were repeated three times.

\section{Determination of Pro content.}

Free proline content in leaf tissues was measured by colorimetric methods (Bates 1973). Values were referred to Lproline (Sigma, St. Louis) calibration curves. Six entire leaves were used for each measurement. Values represent the average of at least three independent experiments.

\section{Measurement of osmotic potential.}

The osmotic potential of leaf tissues infiltrated with $10 \mathrm{mM}$ $\mathrm{MgCl}_{2}$ or with $0.4 \mathrm{M}$ sucrose was determined by psychrometry. Infiltrated or uninfiltrated leaf tissues were pressed onto filter paper discs which immediately were sealed in C-52 Wescor sample chambers connected to an HR-33 T dew point Wescor microvoltometer. Readings were taken at regular intervals until a stable value was obtained, indicating equilibrium. Voltage readings were referred to a standard curve made from a series of $\mathrm{NaCl}$ solutions.

\section{RNA hybridizations.}

Total RNA was prepared according to Alvarez and associates (1998) and $10 \mu \mathrm{g}$ of each sample was used to monitor transcript accumulation by RNA blot hybridization. AtP5CS1 and AtP5CS2 probes were obtained by polymerase chain reaction (PCR) using specific primers pairs (Ábrahám et al. 2003). DNA probes for the Arabidopsis ProDH and P5CDH genes were PCR amplified with gene-specific primers $5^{\prime}$ ACAGCGATAAAACCGAAACATC-3' and 5'-TAGCCACAG GAAATGTTCTTACC-3' $(\mathrm{ProDH})$, and 5'ATGTTGGAGCA CATGG-3' and 5'-GTGACGAGTTCGTAGG-3' (P5CDH). Northern blots were hybridized with radiolabeled cDNA of $5 \mathrm{~S}$ rRNA as loading control. Signals were detected by PhosporImager and quantified by NIH Image computer software. Experiments were repeated three times.

\section{GUS and LUC activity.}

Plants were grown and infiltrated as described above. GUS expression was monitored in AtP5CS1:GUS or AtP5CS2:GUS plants at 24, 48, 72, and 96 hpi by X-Gluc histochemical staining (Ábrahám et al. 2003). In order to detect bioluminescence signals, transgenic plants carrying the AtP5CS1:LUC or AtP5CS2:LUC constructs were sprayed with $1 \mathrm{mM}$ luciferin solution and light emission was detected by a CCD camera (Visilux Imager, Visitron Systems GmbH, Mannheim, Germany) using $20 \mathrm{~min}$ of integration time. LUC expression was monitored at the same time points as those of GUS. Luminescence images were processed by Metaview 4.5r6 software.

\section{ACKNOWLEDGMENTS}

This work was supported by collaboration grant number ARG-7/99 of OMFB-Hungary/SETCyP-Argentina to M. E. Alvarez and L. Szabados; grants from Fundación Antorchas, SECyT-UNC, and Agencia Nacional de Promoción Científica y Tecnológica (BID 1201/OC-AR PICT 01-10123) to M. E. Alvarez; EU 5th Framework Project OPTI-2, QLRT-2000-00103 to L. Szabados; and OTKA grant no. T029430 and T-032428 to L. Szabados. We thank L. Ortega and E. Taleisnik for help with measurement of osmotic potential, A. Király and C. Mas for technical assistance, J. P. Métraux for eds 5 seeds, J. Dangl for $P$. syringae pv. tomato isolates, and E. Taleisnik and $\mathrm{C}$. Koncz for critical reading of the manuscript.

\section{LITERATURE CITED}

Ábrahám, E., Rigó, G., Székely, G., Nagy, R., Koncz, C., and Szabados, L. 2003. Light-dependent induction of proline biosynthesis by abscisic acid and salt stress is inhibited by brassinosteroid in Arabidopsis. Plant Mol. Biol. 51:363-372.

Alvarez, M. E. 2000. Salicylic acid and the machinery of hypersensitive cell death in disease resistance. Plant Mol. Biol. 44:429-442.

Alvarez, M. E., Pennell, P. I., Meijer, P.-J., Ishikawa, A., Dixon, R., and Lamb, C. 1998. Reactive oxygen intermediates mediate a systemic signal network in the establishment of plant immunity. Cell 92:773-784.

Bates, L. S. 1973. Rapid determination of free proline content for waterstress studies. Plant Soil 39:205-207.

Bent, A. F., Kunkel, B. N., Dahlbeck, D., Brown, K. L., Schimdt, R., Giraudat, J., Leung, J., and Sataskawicz, B. J. 1994. RPS2 of Arabidopsis thaliana: A leucine-rich repeat class of plant disease resistance genes. Science 265:1856-1860.

Bi, Y.-M., Kenton, P., Mur, L., Darby, R., and Draper, J. 1995. Hydrogen peroxide does not function downstream of salicylic acid in the induction of PR protein expression. Plant J. 8: 235-245.

Borsani, O., Valpuesta, V., and Botella, M. A. 2001. Evidence for a role of salicylic acid in the oxidative damage generated by $\mathrm{NaCl}$ and osmotic stress in Arabidopsis seedlings. Plant Physiol. 126:1024-1030.

Delaney, T. P., Uknes, S., Vernooij, B., Friedrich, L., Weymann, K., Negrotto, D., Gaffney, T., Gut-Rella, M., Kessmann, H., Ward, E., and Ryals, J. 1994. A central role of salicylic acid in plant disease resistance. Science 266:1247-1250.

Delauney, A. J., and Verma, D. P. S. 1993. Proline biosynthesis and osmoregulation in plants. Plant J. 4:215-223.

Deuschle, K., Funck, D., Hellmann, H., Daschner, K., Binder, S., and Frommer, W. B. 2001. A nuclear gene encoding mitochondrial Deltapyrroline-5-carboxylate dehydrogenase and its potential role in protection from proline toxicity. Plant J. 27:345-356.

Enyedi, A. J., Yalpani, N., Silverman, P., and Raskin, I. 1992. Localization, conjugation, and function of salicylic acid in tobacco during the hypersensitive reaction to tobacco mosaic virus. Proc. Natl. Acad. Sci. U.S.A. 89:2480-2484.

Gaffney, T., Friedrich, L., Vernooij, B., Negrotto, D., Nye, G., Uknes, S., Ward, E., Kessman, H., and Ryals, J. 1993. Requirement of salicylic acid for the induction of systemic acquired resistance. Science 261:754-756.

Genoud, T., and Métraux, J. P. 1999. Crosstalk in plant cell signaling: structure and function of the genetic network. Trends Plant Sci. 4:503507.

Gilmour, S. J., Audrey, M. S., Salazar, M. P., Everard, J. D., and Thomashow, M. F. 2000. Overexpression of the Arabidopsis CBF3 transcriptional activator mimics multiple biochemical changes associated with cold acclimation. Plant Physiol. 124:1854-1865.

Grant, M. R., Godiard, L., Straube, E., Ashfield, T., Lewald, J., Sattler, A., Innes, R. W., and Dangl, J. L. 1995. Structure of the Arabidopsis RPM1 gene which enables dual-specificity disease resistance. Science 269:843-846.

Hamilton, E.W., 3rd, and Heckathorn, S. A. 2001. Mitochondrial adaptations to $\mathrm{NaCl}$. Complex I is protected by anti-oxidants and small heat shock proteins, whereas complex II is protected by proline and betaine. Plant Physiol. 126:1266-1274.

Hare, P. D., and Cress, W. A. 1997. Metabolic implications of stressinduced proline accumulation in plants. Plant Growth Regul. 21:79103.

Hellmann, H., Funck, D., Rentsch, D., and Frommer, W. B. 2000. Hypersensitivity of an Arabidopsis sugar signaling mutant toward exogenous proline application. Plant Physiol. 122:357-367.

Hu, C.-A., Delauney, A. J., and Verma, D. P. 1992. A bifunctional enzyme (delta 1-pyrroline-5-carboxylate synthetase) catalyzes the first two steps in proline biosynthesis in plants. Proc. Natl. Acad. Sci. U.S.A. 89:9354-9358.

Innes, R. W., Bent, A. F., Kunkel, B. N. Bisgrove, S. R., and Staskawicz, B. J. 1993. Molecular analysis of avirulence gene avrRpt2 and identification of a putative regulatory sequence common to all known Pseudomonas syringae avirulence genes. J. Bacteriol. 175:4859-4869.

Jabs, T., Dietrich, R. A., and Dangl, J. L. 1996. Initiation of runaway cell death in an Arabidopsis mutant by extracellular superoxide. Science 273:1853-1856.

Kishor, P. B., Hong, Z., Miao, G. H., Hu, C., and Verma, D. P. S. 1995. Overexpression of [delta]-Pyrroline-5-Carboxylate Synthetase increases proline production and confers osmotolerance in transgenic plants. Plant Physiol. 108:1387-1394.

Kiyosue, T., Yoshiba, Y., Yamaguchi-Shinozaki, K., and Shinozaki, K. 1996. A nuclear gene encoding mitochondrial proline dehydrogenase, an enzyme involved in proline metabolism, is upregulated by proline but downregulated by dehydration in Arabidopsis. Plant Cell 8:13231335 .

Lamb, C. J., and Dixon, R. A. 1997. The oxidative burst in plant disease resistance. Annu. Rev. Plant Physiol. Plant Mol. Biol. 76:419-422. 
Liu, J., and Zhu, J. K. 1997. Proline accumulation and salt-stress-induced gene expression in a salt-hypersensitive mutant of Arabidopsis. Plant Physiol. 114:591-596.

Mani, S., Van De Cotte, B., Van Montagu, M., and Verbruggen, N. 2002. Altered levels of proline dehydrogenase cause hypersensitivity to proline and its analogs in Arabidopsis. Plant Physiol. 128:73-83.

Maxwell, S. A., and Davis, G. E. 2000. Differential gene expression in p53-mediated apoptosis-resistant vs. apoptosis-sensitive tumor cell lines. Proc. Natl. Acad. Sci. U.S.A. 97:13009-13014.

Mindrinos, M., Katagiri, F., Yu, G.-L., and Ausubel, F. M. 1994. The Arabidopsis thaliana disease resistance gene RPS2 encodes a protein containing a nucleotide-binding site and leucine-rich repeats. Cell 78:1089-1099.

Mittler, R. 2002. Oxidative stress, antioxidants and stress tolerance. Trends Plant Sci. 7:405-410.

Nanjo, T., Kobayashi, M., Yoshiba, Y., Kakubari, Y., Yamaguchi-Shinozaki, K., and Shinozaki, K. 1999. Antisense suppression of proline degradation improves tolerance to freezing and salinity in Arabidopsis thaliana. FEBS (Fed. Eur. Biochem. Soc.) Lett. 461:205-210.

Nawrath, C., and Métraux, J. P. 1999. Salicylic acid induction-deficient mutants of Arabidopsis express $P R-2$ and $P R-5$ and accumulate high levels of camalexin after pathogen inoculation. Plant Cell 11:13931404.

Rate, D. N., and Greenberg, J. T. 2001. The Arabidopsis aberrant growth and death 2 mutant shows resistance to Pseudomonas syringae and reveals a role for NPR1 in suppressing hypersensitive cell death. Plant J. 27:203-211.

Rhodes, D., Handa, S., and Bressan, R. A. 1986. Metabolic changes associated with adaptation of plant cells to water stress. Plant Physiol. 82:890-903

Ritter, C., and Dangl, J. L. 1996. Interference between two specific pathogen recognition events mediated by distinct plant disease resistance genes. Plant Cell 8:251-257.

Roosens, N. H., Willem, R., Li, Y., Verbruggen, I., Biesemans, M., and Jacobs, M. 1999. Proline metabolism in the wild-type and in a salt-tolerant mutant of Nicotiana plumbaginifolia studied by ${ }^{13} \mathrm{C}$-nuclear magnetic resonance imaging. Plant Physiol. 121:1281-1290.

Saradhi, P. P., Alia, Arora S., and Prasad, K. V. 1995. Proline accumulates in plants exposed to UV radiation and protects them against UV induced peroxidation. Biochem. Biophys. Res. Commun. 209:1-5.

Savouré, A., Hua, X.-J., Bertauche, N., Van Montagu, M., and Verbruggen, N. 1997. Abscisic acid-independent and abscisic acid-dependent regulation of proline biosynthesis following cold and osmotic stresses. Mol. Gen. Genet. 254:104-109.

Shinozaki, K., and Yamaguchi-Shinozaki, K. 2000. Molecular responses to dehydration and low temperature: differences and cross-talk between two stress pathways. Curr. Opin. Plant Biol. 3:217-223.

Shirasu, K., Nakajima, H., Rajasekhar, V. K., Dixon, R. A., and Lamb, C. 1997. Salicylic acid potentiates an agonist-dependent gain control that amplifies pathogen signals in the activation of defense mechanisms. Plant Cell 9:261-270.

Singh, K., Foley, R. C., and Onate-Sanchez, L. 2002. Transcription factors in plant defense and stress responses. Curr. Opin. Plant Biol. 5:430436.

Siripornadulsil, S., Traina, S., Verma, D. P., and Sayre, R. T. 2002. Molecular mechanisms of proline-mediated tolerance to toxic heavy metals in transgenic microalgae. Plant Cell 14:2837-2847.

Smirnoff, N., and Cumbes, Q. J. 1989. Hydroxyl radical scavenging activity of compatible solutes. Phytochemistry 28:1057-1060.

Strizhov, N., Ábrahám, E., Ökrész, L., Blickling, S., Zilberstein, A., Schell, J., Koncz, C., and Szabados, L. 1997. Differential expression of two P5CS genes controlling proline accumulation during salt-stress requires $\mathrm{ABA}$ and is regulated by ABA1, ABI1 and AXR2 in Arabidopsis. Plant J. 12:557-569.

Tao, Y., Xie, Z., Chen, W., Glazebrook, J., Chang, H. S., Han, B., Zhu, T. Zou, G., and Katagiri, F. 2003. Quantitative nature of Arabidopsis responses during compatible and incompatible interactions with the bacterial pathogen Pseudomonas syringae. Plant Cell 15:317-330.

van Wees, S. C., and Glazebrook, J. 2003. Loss of non-host resistance of Arabidopsis NahG to Pseudomonas syringae pv. phaseolicola is due to degradation products of salicylic acid. Plant J. 33:733-742.

Verbruggen, N., Hua, X. J., May, M., and Van Montagu, M. 1996. Environmental and developmental signals modulate proline homeostasis: evidence for a negative transcriptional regulator. Proc. Natl. Acad. Sci. U.S.A. 93:8787-8791.

Whalen, M. C., Innes, R. W., Bent, A. F., and Staskawicz, B. J. 1991. Identification of Pseudomonas syringae pathogens of Arabidopsis and a bacterial locus determining avirulence on both Arabidopsis and soybean. Plant Cell 3:49-59.

Xin, Z., and Browse, J. 1998. eskimol mutants of Arabidopsis are constitutively freezing-tolerant. Proc. Natl. Acad. Sci. U.S.A. 95:7799-7804.

Yoshiba, Y., Kiyosue, T., Katagiri, T., Ueda, H., Mizoguchi, T., YamaguchiShinozaki, K., Wada, K., Harada, Y., and Shinozaki, K. 1995. Correlation between the induction of a gene for delta 1-pyrroline-5-carboxylate synthase and the accumulation of proline in Arabidopsis thaliana under osmotic stress. Plant J. 7:751-760.

Zhang, C. S., Lu, Q., and Verma, D. P. S. 1995. Removal of feedback inhibition of delta 1-pyrroline-5-carboxylate synthase, a bifunctional enzyme catalyzing the first two steps of proline biosynthesis in plants. $J$ Biol. Chem. 270:20491-20496. 